\title{
Performance of Digital Silicon Photomultipliers for Time of Flight PET Scanners
}

\author{
R. Marcinkowski, S. España, H. Thoen, S. Vandenberghe
}

\begin{abstract}
The performance of Digital Silicon Photomultipliers (dSiPM) coupled to a LYSO array containing $15 \times 15$ pixels with a size of $2 \times 2 \times 22 \mathrm{~mm}^{3}$ is evaluated to determinate their potential for whole body Time of Flight (TOF) PET scanners.

The detector pixels are smaller in size than the light sensors and therefore light spreading is required to determine the crystal where interaction occurred. A light guide of $1 \mathrm{~mm}$ was used to spread the light and neighbor logic (NL) configuration were employed to ensure correct crystals identification. We studied the energy resolution and coincidence resolving time (CRT) for different trigger levels. The measured average energy resolution across detector was $14.5 \%$. Prior to measurements of time resolution skew time calibration of $\mathrm{dSiPM}$ was performed. The average CRT achieved using trigger level 1 option was 376 ps FWHM. Finally, we studied the amount of events that are disregarded due to dark count effects for different trigger levels and temperatures. Our studies show that a trade-off must be made between the detector's CRT and sensitivity due to its vulnerability to dark counts. To employ dSiPM in TOF PET systems without 1:1 coupling effective cooling is necessary to limit dark count influence.
\end{abstract}

\section{INTRODUCTION}

S ILICON Photomultipliers (SiPMs) are solid-state $S_{\text {single-photon sensitive devices made of arrays of Geiger- }}$ mode avalanche photodiodes. Analog SiPMs combine the pulses by multiple photon detections into one analog output signal, but this step requires complex electronics vulnerable to noises.

Philips Digital Photon Counting (PDPC) has introduced a novel type of SiPM called digital silicon photomultiplier (dSiPM) [1-4]. These devices integrate CMOS electronics into the SiPM chip for every microcell, resulting in a fully digital readout. By integrating low-power CMOS digital electronics into the silicon photomultiplier chip, each photon detection is converted into a high-speed digital pulse that can be directly counted by on-chip counter circuitry. As a result, they achieve gain-independent photon counting with accurate photon arrival time information, which are important factors for medical imaging applications. In particular, these devices can be used for PET and SPECT imaging and do have benefits in spatial, timing and energy resolution.

In this work we present a performance study of dSiPM devices together with pixelated LYSO arrays with $2 \mathrm{~mm}$

Manuscript received November 16, 2012. This work was supported in part by EU FP7 project SUBLIMA, Grant Agreement No. 241711, see also www.sublima-pet-mr.eu.

The authors are with MEDISIP, Ghent University - iMinds-IBiTech, ELIS department, De Pintelaan 185, Block B, B-9000 Ghent, Belgium (telephone: +32 933242 36, email: radoslaw.marcinkowski@ugent.be, web: http://medisip.elis.ugent.be) transverse pixel size for their use in whole body TOF-PET systems. The crystal height was $22 \mathrm{~mm}$ to enable high stopping power and sensitivity necessary for whole body imaging. This size of the crystals was chosen according to a scanner design study based on GATE Monte Carlo simulations, which has been performed in our group. For the simulations, a cylindrical phantom of $35 \mathrm{~cm}$ in diameter and axial length of $12 \mathrm{~cm}$ filled with water was used. The phantom contained three rings of $5 \mathrm{~mm}$ diameter hot spheres, ten spheres per ring, located 5,10 and $15 \mathrm{~cm}$ from the center. As presented in Table I, the use of $2 \mathrm{~mm}$ crystal transverse pixel size compared to $4 \mathrm{~mm}$ improves spatial resolution in each direction by $30 \%$. Ordered subsets expectation maximization (OSEM) algorithm, with 10 subsets and 10 iterations, was used for image reconstruction. Listmode data was used as algorithm's input. No PSF modeling was done inside reconstruction.

TABLE I. SPATIAL RESOLUTION@CFOV FOR DIFFERENT PIXEL SIZE

\begin{tabular}{cccc}
\hline $\begin{array}{c}\text { Pixel size } \\
{\left[\mathrm{mm}^{3}\right]}\end{array}$ & $\begin{array}{c}\text { Radial } \\
{[\mathrm{mm}]}\end{array}$ & $\begin{array}{c}\text { Tangential } \\
{[\mathrm{mm}]}\end{array}$ & $\begin{array}{c}\text { Axial } \\
{[\mathrm{mm}]}\end{array}$ \\
\hline $2 \times 2 \times 22$ & 2.2 & 2.2 & 2.4 \\
$4 \times 4 \times 22$ & 3.3 & 3.4 & 3.5 \\
\hline
\end{tabular}

\section{MATERIALS AND METHODS}

A single dSiPM detector module, called a tile, is an array built from 16 independent sensors called dies. Dies are arranged in a $4 \times 4$ matrix, which results in an outer dimension of a tile equal to $32 \mathrm{~mm} \times 32 \mathrm{~mm}$. Between vertical rows of dies there are $800 \mu \mathrm{m}$ wide dead areas of the detector. Each die is further divided into $4 \mathrm{SiPM}$ pixels, arranged in a $2 \times 2$ matrix and with overall $4 \mathrm{~mm}$ pitch. Each pixel consists 3200 cells (i.e. avalanche photodiodes) for dSiPM type DPC-320044-22. Readout of the fired cells and timestamp generation is performed on the level of the individual die.

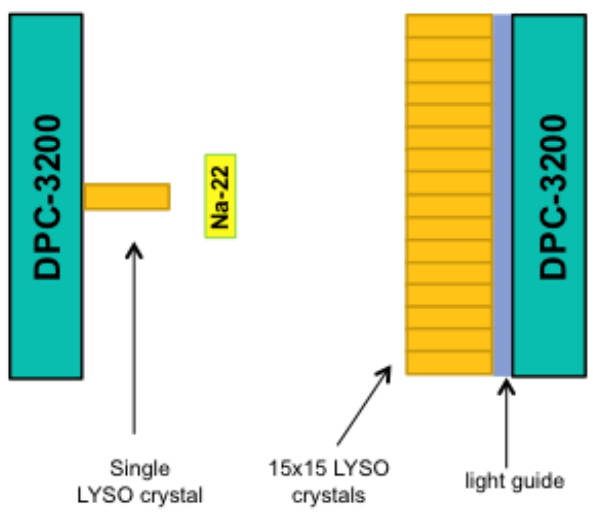

Fig. 1. Schematic of the measurements setup. 
One to one coupling between LYSO crystals with $2 \mathrm{~mm}$ pitch and dSiPM pixels with $4 \mathrm{~mm}$ pitch was not possible. Therefore, in order to achieve correct crystal identification, a light guide was used to allow light sharing among different $\mathrm{dSiPM}$ pixels. In addition, the neighbor logic (NL) feature of the dSiPM was used. Neighbor logic is a configuration option of dSiPM that allows the first successfully triggered and validated die to force all others dies to acquire and process data, bypassing their own trigger and validation routines. That allows to record for each event the dies that collected only a small fraction of light, which in normal acquisition mode (not using NL) would be insufficient to fulfill validation thresholds.

Fig. 1 shows a schematic of the experimental setup that was used on this study. Two dSiPM tiles (type DPC-3200-4422) were placed at $16 \mathrm{~cm}$ distance face to face for the measurements. One device was optically coupled to a $1 \mathrm{~mm}$ thick light guide made of borosilicate glass and to a LYSO array containing $15 \times 15$ pixels with size of $2 \times 2 \times 22 \mathrm{~mm}^{3}$ and white reflector with $0.05 \mathrm{~mm}$ thickness. The other device was directly coupled to a single LYSO pixel with size of $2 \times 2 \times 22 \mathrm{~mm}^{3}$ and wrapped with Teflon tape placed at the center of one of dSiPM pixels. We used silicone optical grease,BC-630 (Saint-Gobain) for the optical coupling. A ${ }^{22} \mathrm{Na}$ point source with activity of $30 \mu \mathrm{Ci}$ was used in all the measurements. Data acquisition was performed using the Philips Digital Photon Counter Technology Evaluation Kit (PDPC-TEK) connected to a PC. We used the coincidence acquisition mode in all measurements presented in this report. Fan-ventilators were placed at the back of each dSiPM tile in order to dissipate the heat produced by the processing boards and the whole setup was introduced in a temperature chamber with internal temperature set to $2^{\circ} \mathrm{C}$. The working temperature of the devices during measurements was held within $6^{\circ} \mathrm{C}$ to $7.5^{\circ} \mathrm{C}$.

For all conducted studies we used a fixed dSiPM configuration of validation level 8 (minimal amount of fired cells to recognize recorded event as valid) and validation time of $40 \mathrm{~ns}$ (a time during which desired number of cells for validation must be acquired). Integration time (a period of time during which number of fired cells is counted,) was set to $165 \mathrm{~ns}$ and a coincidence window was set to $20 \mathrm{~ns}$. NL was used in for all measurements.

We performed energy and timing resolution measurements for different configuration setups of the dSiPM acquisition. In particular we conducted studies of trigger level influence on coincidence resolving time (CRT) of dSiPM.

\section{RESULTS}

\section{A. Crystal Identification}

The resulting flood map is shown in Fig. 2 where all the crystals can be clearly identified. The flood map was obtained for trigger level 1, validation level 8, integration time $165 \mathrm{~ns}$ and validation time $40 \mathrm{~ns}$. Center of gravity algorithm was used.

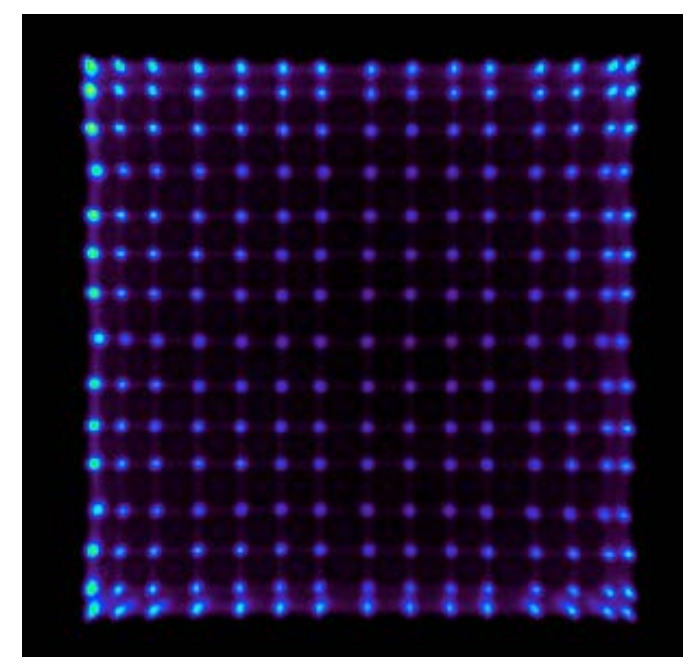

Fig. 2. Flood map of the $15 \times 15$ LYSO array coupled to the dSiPM. Obtained for trigger level 1.

\section{B. Energy Resolution}

Fig. 3 depicts the $511 \mathrm{keV}$ photopeak position across all $15 \times 15$ crystals of the LYSO array coupled to the dSiPM. Average photopeak position at the centers of detector dies is located at 2400 optical photons. Three visible rows of crystals with average photopeak position of 2100 optical photons can be noticed across array. These rows correspond to crystals located directly over the dead area gaps of dSiPM. Shift of photopeak position for those crystals is due to lost optical photons hitting these gaps.

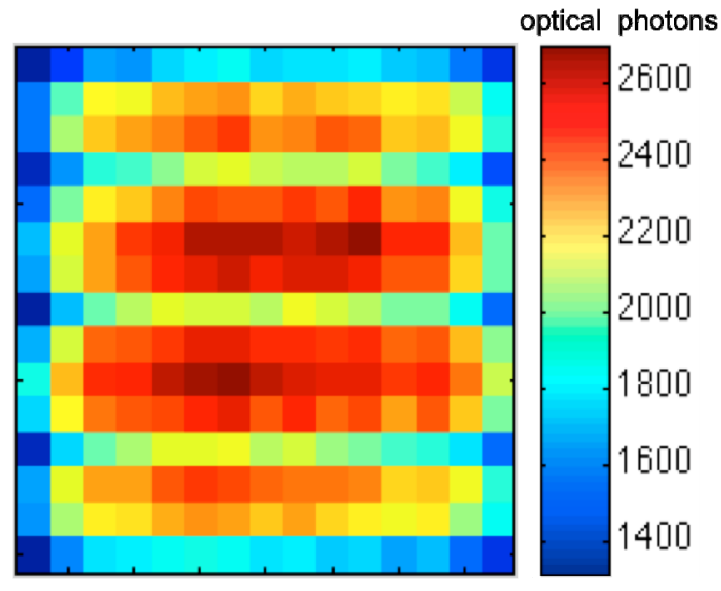

Fig. 3. $511 \mathrm{keV}$ photopeak position across all $15 \times 15$ crystals of the LYSO array.

Fig. 4 depicts the energy resolution at $511 \mathrm{keV}$ across all 225 crystals coupled to the dSiPM. Average energy resolution across the detector yields a FWHM of $14.5 \%$ with sigma equal to $1.3 \%$. No cross talk and saturation corrections were included [5]. 


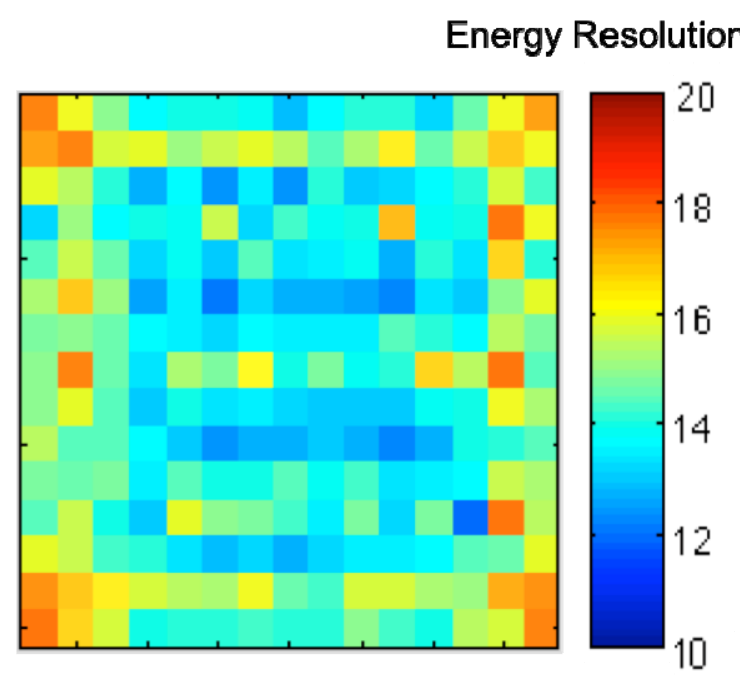

(\%)

Fig. 4. Energy resolution across all 225 crystals of LYSO array.

\section{Timing Resolution}

Due to the fact that the light coming from a single crystal is spread among different dies (each die contains an individual timestamp) it was necessary to conduct a skew time calibration between dies before performing timing resolution measurements. In order to accomplish such calibration we need to record synchronized events, which occurrence time is known, for different dies and compare theirs responses. For that purpose we used the same setup shown in Fig. 1 and acquired coincidence events using configuration with trigger level value 1 . In that way, the detector with a single LYSO serves as a reference time. In the other detector, the pixels from the LYSO array located closer to the center of each die have a very high probability that the first optical photon arrives to that particular die. Therefore, the skew time calibration can be performed by aligning the mean values of the time difference histograms between the detector with a single LYSO crystal and one selected LYSO crystal per die of the other detector. In that way we measured skew time varying from $78 \mathrm{ps}$ to $858 \mathrm{ps}$. Skew time values for individual dies across the detector are presented in Fig. 5. Fig. 6 shows how applying the time skew correction improves the CRT of crystals in the array.

\begin{tabular}{|c|c|c|c|}
\hline 156 & 97 & 858 & 136 \\
\hline 214 & 136 & 819 & 175 \\
\hline 78 & 78 & 682 & 156 \\
\hline 78 & 0 & 702 & 175 \\
\hline
\end{tabular}

Fig. 5. Time skew between dies in [ps]. Die with value of 0 was chosen as reference for calculations.

Fig. 7 depicts the CRT obtained for all the crystals in the array for trigger level 1 . The average CRT across the dSiPM was 376 ps. It can be noticed that the CRT for the crystals located directly over the dead areas is degraded compared to other crystals.

\section{Influence of Trigger Level on Timing Resolution}

We investigated how the different trigger levels change timing resolution of the detector. Fig. 8 presents histograms of crystals CRT for different trigger level. It can be clearly observed how significant CRT degradation occurs with increasing dSiPM trigger level. The average CRT across the detector increases to $558 \mathrm{ps}$ for trigger level two, and to $758 \mathrm{ps}$ and $1483 \mathrm{ps}$ for trigger level three and four respectively.

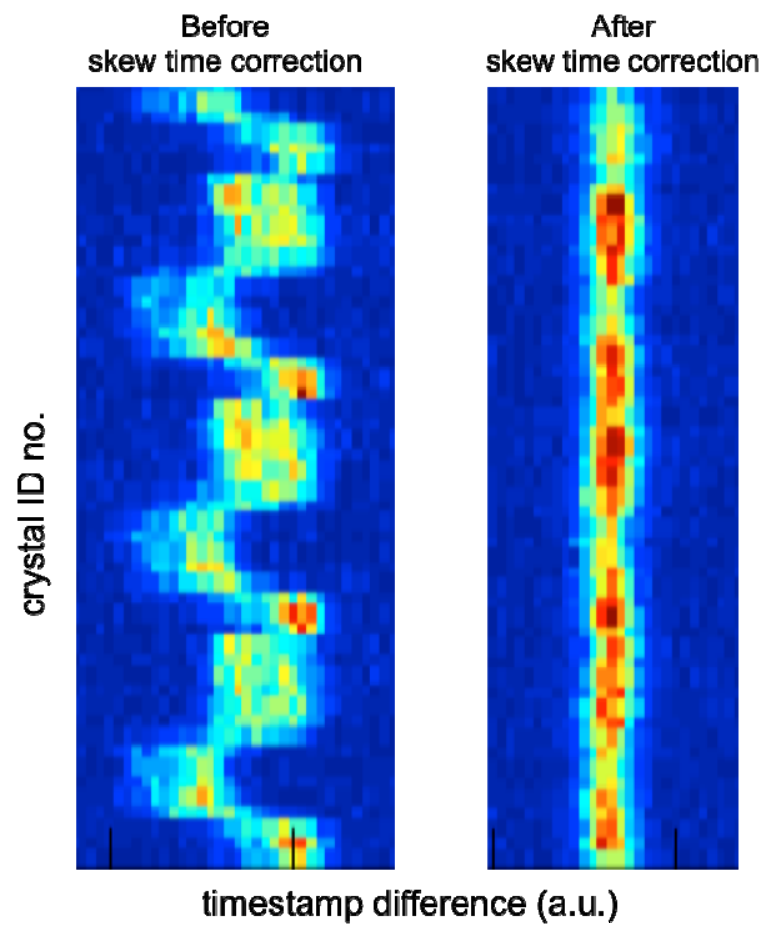

Fig. 6. Histograms (every row of figure) of the timestamp difference between reference single LYSO crystal and individual crystal in the LYSO array before applying the skew correction (left) and after applying the skew correction (right).

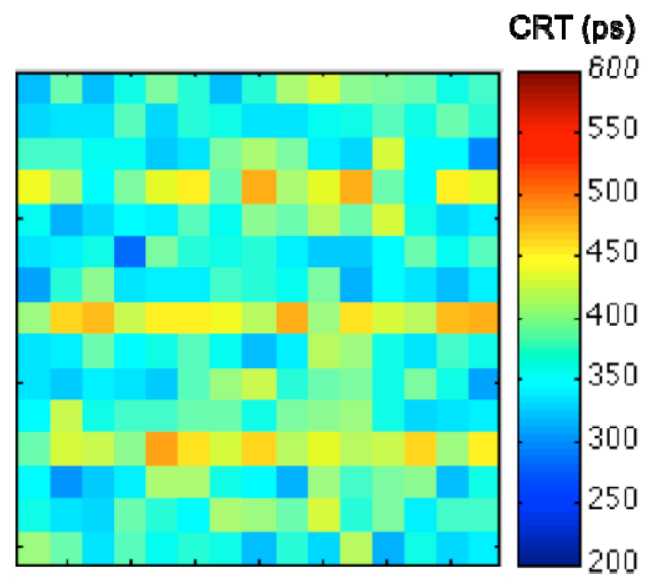

Fig. 7. Timing resolution across all crystals of LYSO array. Measured with dSiPM configuration of trigger lv.1, validation lv. 8, integration time of 165 $\mathrm{ns}$, validation time of $40 \mathrm{~ns}$, NL was used. 


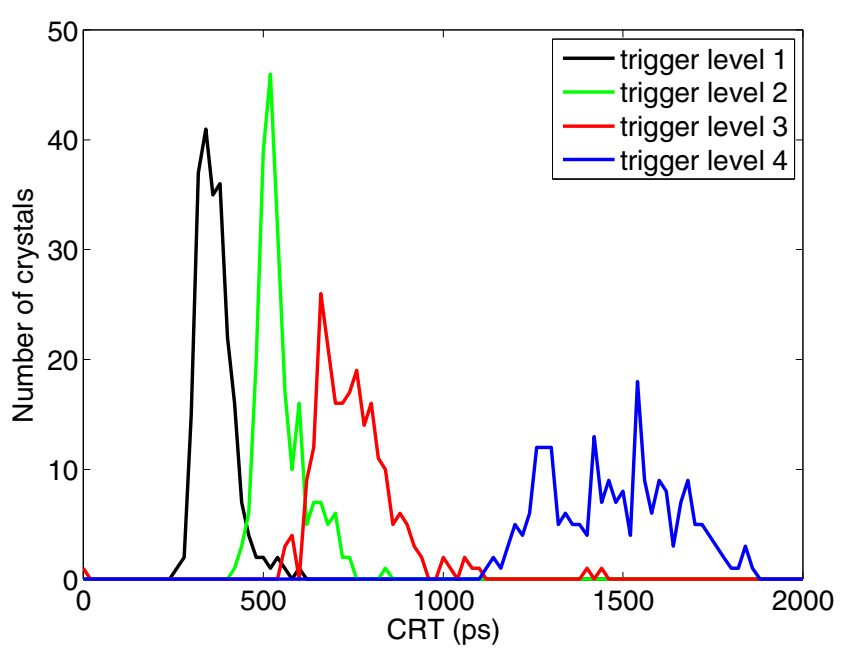

Fig. 8. Histogram of the CRT for all crystals in the LYSO array using different trigger levels. All measurements were conducted for validation lv. 8, integration time of $165 \mathrm{~ns}$ and validation time of $40 \mathrm{~ns}$, NL was used.

\section{E. Valid Events}

As presented in the previous section, to achieve the best CRT it is necessary to work with trigger level 1 . However working at this level of triggering means that detector is strongly vulnerable to the influence of dark counts because every single dark count will trigger the acquisition sequence of the die and no new events can be measured during recharge routine at the end of that sequence. The acquisition sequence of the dSiPM is presented in Fig. 9.

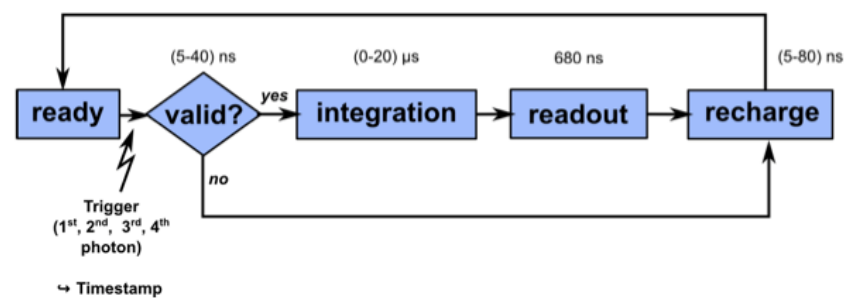

Fig. 9. Schematic of acquisition sequence of dSiPM.

Those events will be normally discarded inside validation step and the die will be recharged. During the recharging process, the die is not able to record new events so if a different die is validated during that time, the information from the recharging die will not be available. If that situation occurs in a die located close to the crystal emitting the light, an important fraction of optical photons would be lost. The probability of such situation depends on the trigger level set for the acquisition and the dark count rate, which depends on the temperature. In addition, the crystals at the center of the detector share the light into more dies increasing the probability of losing a fraction of the light compared to crystals closer to the edges. This effect can be observed in Fig 2 where the crystals on the edges are more intense than the ones in the center of the array.

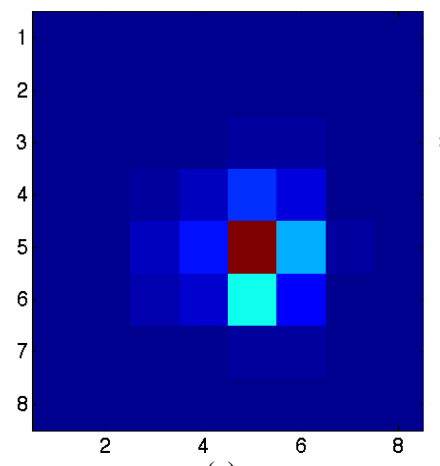

(a)

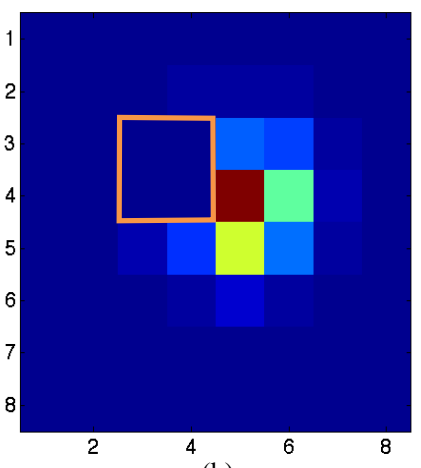

(b)
Fig. 10. Examples of recorded events where all dies were available during photon detection (a) where one die (orange box) adjacent to the place of gamma interaction was lost (b). Color scale represents number of recorded optical photons in dSiPM's pixels $(8 \times 8)$.

Fig. 10 (a) shows one event where all dies were recorded together while Fig. 10 (b) shows one event where one die was lost due to the process previously explained. It can be noticed that an important fraction of the light is lost in this last scenario. Using such kind of events will result in a degradation of the energy resolution and crystal identification. For this reason, in all presented results of this study we discarded all the events, when at least one die adjacent to the crystal in which gamma interaction took place was missing.

Table II shows the percentage of accepted events from the total amount of recorded events for both singles and coincidences events. Values for different trigger levels are shown. Table III shows the values obtained for acquisitions performed at room temperature where the dark count rate was much higher. It can be observed that the percentage of accepted event decrease for lower trigger levels and higher temperature.

TABLE II. PERCENTAGE OF ACCEPTED EVENT @ 6 -7.5 C.

\begin{tabular}{ccc} 
Trigger level & singles & coincidences \\
\hline 1 & $39 \%$ & $16 \%$ \\
2 & $66 \%$ & $44 \%$ \\
3 & $69 \%$ & $48 \%$ \\
4 & $80 \%$ & $64 \%$ \\
\hline
\end{tabular}

TABLE III. PERCENTAGE OF ACCEPTED EVENT @ $20-22^{\circ} \mathrm{C}$.

\begin{tabular}{ccc} 
Trigger level & singles & coincidences \\
\hline 1 & $11 \%$ & $1 \%$ \\
2 & $37 \%$ & $14 \%$ \\
3 & $39 \%$ & $15 \%$ \\
4 & $68 \%$ & $41 \%$ \\
\hline
\end{tabular}

\section{CONCLUSION}

We made an evaluation of the performance of dSiPM coupled to a $15 \times 15$ pixelated LYSO array with $2 \times 2 \times 22 \mathrm{~mm}^{3}$, pixel size for TOF-PET scanners. Due to the lack of one-toone coupling, a light guide was needed for correct crystal identification along with the use neighbor logic feature of the $\mathrm{dSiPM}$. The measured average energy resolution across the 
detector was $14.5 \%$. The average CRT achieved using trigger level 1 option was 376 ps FWHM.

Our study shows that a trigger level 1 or 2 must be used to obtain CRT values similar to state of the art TOF-PET scanners. However, due to the light sharing needed for correct crystal identification, the fraction of valid events is strongly dependent on the dark count rate and the trigger level. Therefore, a trade-off between CRT and fraction of valid events has to be done. The fraction of accepted events in low trigger level acquisition mode can be increased by measuring at a lower temperature in order to reduce the dark count rate.

\section{REFERENCES}

[1] Th. Franch et al., Nuclear Science Symposium Conference Record, N28-5. 2009

[2] Th. Franch et al., Nuclear Science Symposium Conference Record, N58-1, 2010

[3] C. Degenhardt et al., Nuclear Science Symposium Conference Record, J04-1, 2009

[4] C. Degenhardt et al., "Array of Digital Silicon Photomultipliers Intrinsic Performance and Application to Scintillator Readout", Nuclear Science Symposium Conference Record, 2010

[5] T. van Dam et al.,"The statistical distribution of the number of counted scintillation photons in digital silicon photomultipliers: model and validation", Phys. Med. Biol. 57 4885-4903, 2012 